\title{
Does less surgical trauma result in better outcome in management of iatrogenic tracheobronchial laceration?
}

\author{
Dominik Herrmann ${ }^{1}$, Jan Volmerig ${ }^{1}$, Ahmad Al-Turki ${ }^{1}$, Monique Braun ${ }^{1}$, Anke Herrmann ${ }^{2}$, Santiago Ewig ${ }^{3}$, \\ Erich Hecker ${ }^{1}$ \\ ${ }^{1}$ Thoraxzentrum Ruhrgebiet, Department of Thoracic Surgery, Evangelisches Krankenhaus, Herne, Germany; ${ }^{2}$ Institute of Virology, University of \\ Duisburg-Essen, Essen, Germany; ${ }^{3}$ Thoraxzentrum Ruhrgebiet, Department of Respiratory and Infectious Diseases, Evangelisches Krankenhaus, \\ Herne, Germany \\ Contributions: (I) Conception and design: E Hecker, S Ewig, D Herrmann; (II) Administrative support: E Hecker, S Ewig, J Volmerig; (III) Provision \\ of study materials or patients: E Hecker, J Volmerig; (IV) Collection and assembly of data: D Herrmann, A Al-Turki, M Braun, A Herrmann; (V) \\ Data analysis and interpretation: D Herrmann, A Herrmann; (VI) Manuscript writing: All authors; (VII) Final approval of manuscript: All authors. \\ Correspondence to: Erich Hecker, MD. Hordeler Straße 7-9, 44651 Herne, Germany. Email: e.hecker@evk-herne.de.
}

Background: Iatrogenic tracheobronchial injury is a rare, but severe complication of endotracheal intubation. Risk factors are emergency intubation, percutaneous dilatational tracheostomy and intubation with double lumen tube. Regarding these procedures, underlying patients often suffer from severe comorbidities. The aim of this study was to evaluate the results of a standardized treatment algorithm in a referral center with focus on the surgical approach.

Methods: Sixty-four patients with iatrogenic tracheal lesion were treated in our department by standardized management adopted to clinical findings between 2003 and 2019. Patients with superficial laceration were treated conservatively. In the case of transmural injury of the tracheal wall and necessity of mechanical ventilation, patients underwent surgery. We decided on a cervical surgical approach for lesions limited to the trachea. In case of involvement of a main bronchus we performed thoracotomy. Data were evaluated retrospectively.

Results: In 19 patients the tracheal lesion occurred in elective intubation and in 17 patients during emergency intubation. In 23 cases a tracheal tear occurred during percutaneous dilatational tracheostomy and in three patients at replacement of a tracheostomy tube. Two patients received laceration during bronchoscopy. Twenty-nine patients underwent surgery with cervical approach and 14 underwent thoracotomy. There was no difference in the mortality of these groups. Treatment of tracheal tear was successful in 62 individuals. Nine patients died of multi organ dysfunction syndrome (MODS), two of them during surgery.

Conclusions: Iatrogenic tracheal laceration is a life-threatening complication and the mortality after tracheal injury is high, even in a specialized thoracic unit. Conservative management in patients with superficial tracheal lesion is a feasible procedure. In case of complete laceration of tracheal wall, surgical therapy is recommendable, whereby several approaches of surgical management seem to be equivalent.

Keywords: Iatrogenic tracheal injury; tracheal surgery; intubation

Submitted Jul 17, 2019. Accepted for publication Sep 27, 2019.

doi: $10.21037 /$ jtd.2019.10.22

View this article at: http://dx.doi.org/10.21037/jtd.2019.10.22

\section{Introduction}

Tracheal laceration is a seldom, but severe complication of endobronchial intubation. The incidence of tracheal injury, dependent on the manner of intubation is approximately $0.005 \%$ in a single-lumen intubation (1), 0.05-0.19\% in a double-lumen intubation (2) and $0.2-0.7 \%$ after 
Table 1 Proposal for classification of postintubation tracheal lesion made by Cardillo et al. (10) in 2010

\begin{tabular}{ll}
\hline Level & Mucosal or submucosal tracheal involvement without mediastinal emphysema or oesophageal injury \\
\hline PITL I & $\begin{array}{l}\text { Tracheal lesion up to the muscular wall with subcutaneous or mediastinal emphysema without oesophageal injury or } \\
\text { mediastinitis }\end{array}$ \\
PITL IIIA & $\begin{array}{l}\text { Complete laceration of the tracheal wall with oesophageal or mediastinal soft-tissue hernia without oesophageal } \\
\text { injury or mediastinitis }\end{array}$ \\
PITL IIIB & Any laceration of the tracheal wall with oesophageal injury or mediastinitis \\
\hline
\end{tabular}

PITL, post-intubation tracheal laceration.

percutaneous dilatational tracheostomy (3).

Dyspnoea, subcutaneous or mediastinal emphysema and hemoptysis are typical signs and symptoms of tracheal injury and may occur delayed. Clinically apparent injuries are often life threatening and patients are in need of rapid and experienced evaluation and treatment.

The optimal management of iatrogenic tracheal laceration remains a subject of debate and several proposals were published during the last years (4-6). Common options for the treatment of iatrogenic tracheal tears vary from conservative management to surgery, dependent on depth of the lesion, clinical status and necessity of further mechanical ventilation.

Successful stenting of the tracheobronchial injury is reported in a number case reports and single-centerexperiences $(5,7,8)$ as well as different surgical approaches $(9)$. Unfortunately, criteria of decision-making are inconsistent and evidence from randomised trials or at least large observational studies is still missing.

The aim of this study was to evaluate the results of a standardized management of iatrogenic tracheal injury in a thoracic surgical referral center according to a widely accepted classification (10) with focus on the surgical approach.

\section{Methods}

Our study population includes all patients with an iatrogenic tracheal tear treated in our department between May 2003 and May 2019. All patients showed a longitudinal laceration of the paries membranaceous.

Depending on the site of tracheal injury, the necessity of mechanical ventilation and the post-intubation tracheal laceration (PITL)-level, these patients were treated by surgery or interventional bronchoscopy. We applied the PITL-classification proposed by Cardillo et al. (10) (Table 1).
The treatment algorithm is shown in Figure 1.

Diagnosis and grading of tracheal laceration were confirmed by bronchoscopy and esophagoscopy.

Patients with tracheal injury in PITL-level I or II were always treated conservatively under repetitive bronchoscopic surveillance, in PITL II additionally with application of fibrin sealant. In individuals with PITL IIIAlesion, treatment decision was based on the necessity of mechanical ventilation and clinical conditions.

Patients with tracheal laceration in PITL-level IIIB were treated by surgery.

In individuals with injury limited to the trachea we used a transcervical approach, in cases including injury of a main bronchus we performed thoracotomy.

All patients received a single shot i.v. antibiotic prophylaxis with two gr of cefazolin. Patients with tracheal injury in PITL-level III inhaled with $80 \mathrm{mg}$ tobramycin twice daily for one week (11) and received broad-spectrum antibiotics for seven days in case of mediastinitis.

\section{Interventional techniques}

In case of PITL-level I and II conservative management with repetitive bronchoscopy was performed. Fibrin sealant (Tissucol $^{\circledR}$, Baxter, Deerfield, MA, USA) was applied onto the tear in cases of PITL-level II and PITL IIIA when no mechanical ventilation was required (Figure 2).

In patients with PITL IIIA and necessity of further mechanical ventilation and in individuals with PITL IIIB we performed surgical repair of the tracheal tear.

In patients with isolated injury of trachea in PITL-level III a transcervial approach was used, which is described in detail by Angelillo-Mackinlay (9). In case of laceration of the distal trachea, we used a bronchoscope for visualisation and endoscopic instruments for suture of the lesion in the membranous part. A single-lumen tube or jet ventilation 


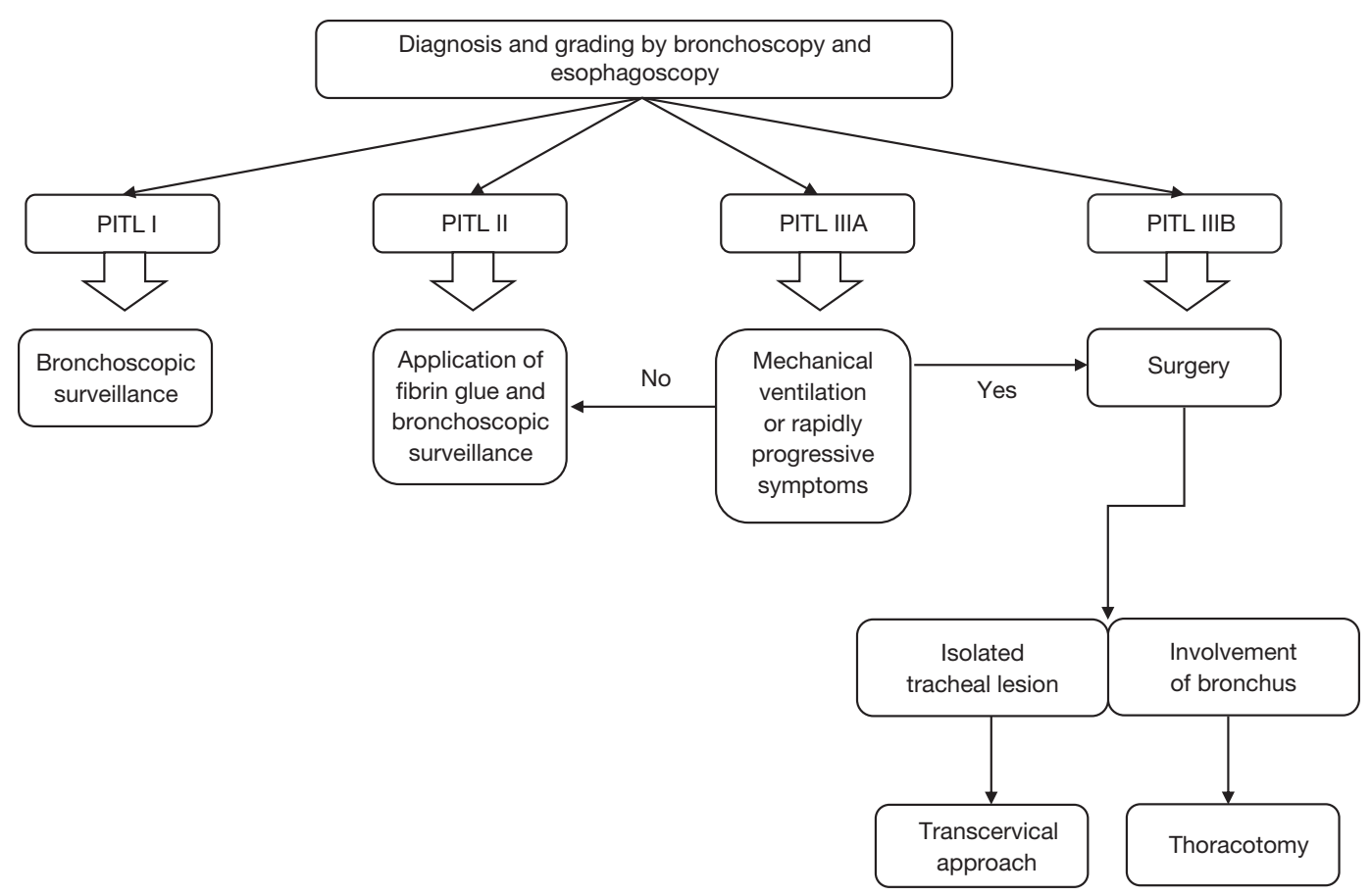

Figure 1 Proposed treatment algorithm for management of iatrogenic tracheal laceration.
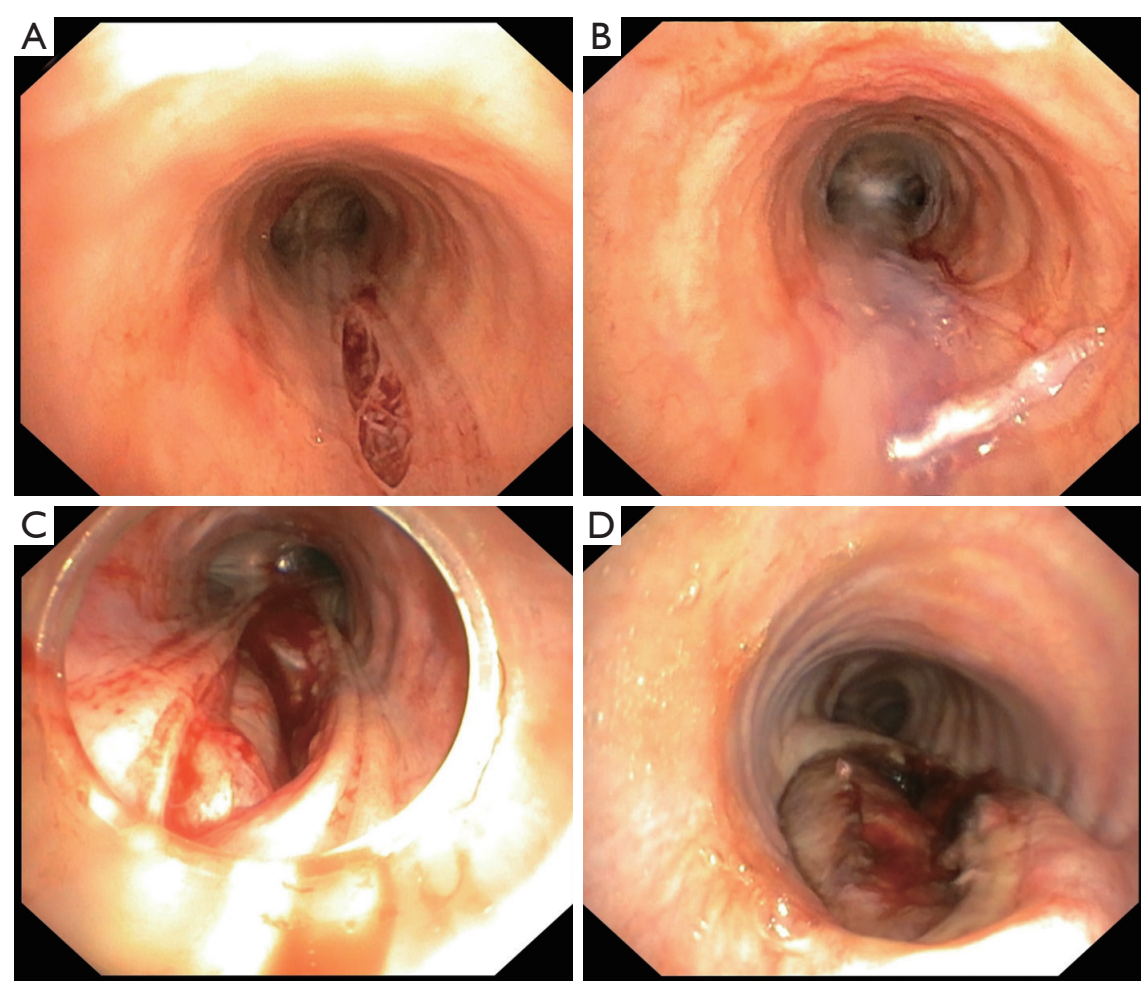

Figure 2 Different types of PITL-levels. (A) PITL-level II in middle third of trachea; (B) fibrin sealant of tracheal lesion in the left picture; (C) tracheal injury in PITL-level IIIA; (D) tracheal laceration in PITL-level IIIB with oesophageal injury. PITL, post-intubation tracheal laceration. 

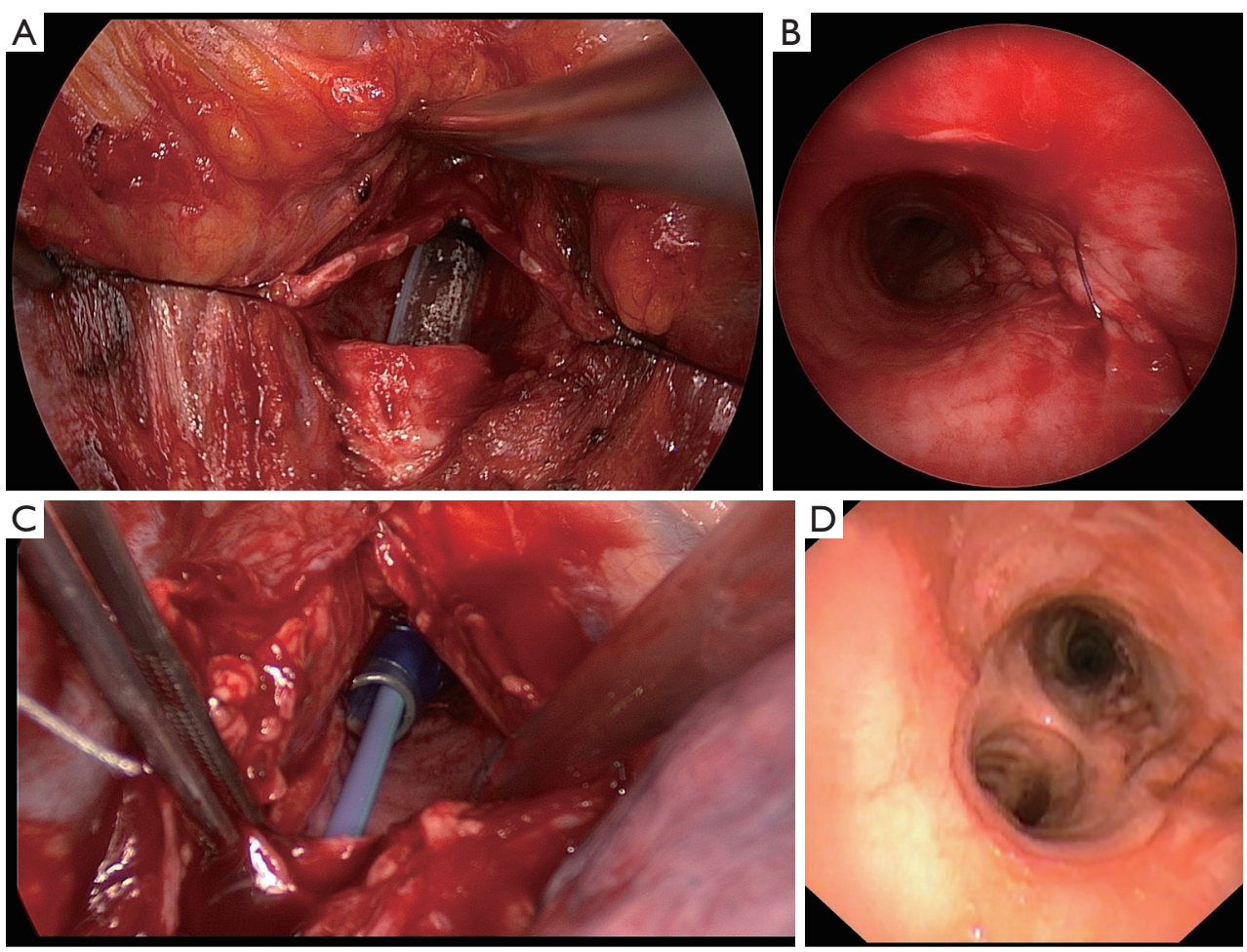

Figure 3 Intraoperative view and postoperative results. (A) Transcervical approach of an isolated injury of the trachea; (B) bronchoscopic image after surgery of the laceration at the left picture in first row; (C) the operative field in right sided thoracotomy for a tracheal injury including right main bronchus. Patient's ventilation via jet ventilation; (D) bronchoscopic image after surgery of the lesion at left picture in second row.

was used for ventilation. We placed a chest tube only in case of pleural fluid at the affected side.

In patients with injury PITL-level III involving a main bronchus surgical approach was thoracotomy on the side of the affected main bronchus (Figure 3). Thoracotomy was performed in lateral decubitus position under one-lungventilation with corresponding double-lumen intubation. Suture of the tracheal injury was executed in double running technique. When possible, the esophagus was used to enforce the suture. The reconstructed trachea was covered by esophagus, pericardial fat, pericardium or intercostal muscle flap. After thoracotomy we placed a chest tube near the sealed laceration.

Regardless of the approach, we performed a mediastinal debridement and irrigation of mediastinum with a povidone-iodine solution.

\section{Postoperative care}

All patients were observed at intensive care unit after operation.

The suture was usually checked twice, at the first and second day after surgery.

In patients who required mechanical ventilation after intervention, the cuff of the tube was placed distal of the laceration if possible. The positive end-expiratory pressure and tidal volume were kept as low as possible ensuring sufficient oxygenation and ventilation.

\section{Data recording and statistics}

Medical records were analyzed retrospectively. Data were completely available for all patients. Analyses were conducted after removal of patient identifying information.

Data entry was carried out using Microsoft Excel 2016. For descriptive statistical assessment, SPSS for windows Version 23.0 was used. Wilcoxon-Mann-Whitney test and Kruskal-Wallis rank test were used for comparison of two quantitative variables. Kruskal-Wallis Test was followed by Dunn's post-hoc test. Fisher's exact test was used for 
Table 2 Patients' characteristics

\begin{tabular}{|c|c|}
\hline Variable & All cases $(n=64)$ \\
\hline Age (years) & $64.77(13.5)$ \\
\hline \multicolumn{2}{|l|}{ Gender } \\
\hline Female (n, \%) & $47(73.4)$ \\
\hline Male (n, \%) & $17(26.6)$ \\
\hline Hospitalisation (days) & $8(5-12)$ \\
\hline BMI $\left(\mathrm{kg} / \mathrm{m}^{2}\right)$ & $29(26-31)$ \\
\hline Referred to our institution (n, \%) & $48(75.0)$ \\
\hline Length of injury (cm) & $4(3-5)$ \\
\hline Surgical treatment $(\mathrm{n}, \%)$ & $43(67.2)$ \\
\hline \multicolumn{2}{|l|}{ PITL } \\
\hline $\mathrm{I}(\mathrm{n}, \%)$ & $2(3.1)$ \\
\hline II $(\mathrm{n}, \%)$ & $14(21.9)$ \\
\hline IIIA (n, \%) & $28(43.8)$ \\
\hline IIIB (n, \%) & $20(31.3)$ \\
\hline CRP before treatment (mg/dL) & $6.58(6.5)$ \\
\hline Leukocyte count before treatment $\left(10^{3} / \mu \mathrm{L}\right)$ & $14.75(8.48)$ \\
\hline 30-day mortality (n, \%) & $9(14.1)$ \\
\hline 90-day mortality (n, \%) & $9(14.1)$ \\
\hline In-hospital mortality (n, \%) & $10(15.6)$ \\
\hline
\end{tabular}

$\mathrm{SD}$, standard deviation; BMI, body mass index; CRP, C-reactive protein.

comparison of categorical variables. Continuous variables are presented as means with standard deviation (SD) or median with interquartile range. Categorical data are reported as counts and percentages. Statistical significance was set at a $P$ value less than 0.05 .

Institutional Review Board was consulted. No approval was required, because of retrospectively and anonymously data analysis and interpretation, as indicated by our Institutional Review Board. Written informed consent from the patients was waived due to retrospective nature of the study.

\section{Results}

Sixty-four patients with tracheal injury following intubation were treated in our department between May 2003 and April 2019. Patients' characteristics are described in Table 2.

The injury occurred in 19 patients during elective intubation $(29.7 \%)$ and in 17 patients during emergency intubation $(26.6 \%)$. In 23 cases the laceration occurred in percutaneous dilatational tracheostomy $(35.9 \%)$ and in 3 patients at replacement of a tracheostomy tube (4.7\%). Two patients underwent bronchoscopy, when the tracheal lesion occurred (3.1\%). Only 7 injuries took place during intubation with a double-lumen tube, in 29 cases the injury occurred during intubation with single-lumen tube.

There was an isolated lesion of the trachea in 49 patients. In 15 cases the laceration involves one of the main bronchi as well.

In 2 patients $(3.1 \%)$ with PITL level I the lesion was an incidental finding during routine bronchoscopy after lung resection. Fourteen patients (21.9\%) presented with a split in PITL level II, while $28(43.8 \%)$ had a laceration according to PITL level IIIA. Twenty patients (31.3\%) presented with transmural laceration and mediastinitis and were classified PITL level IIIB.

Forty-three patients were treated by surgery and 21 patients were treated only by bronchoscopic fibrin glue application. Five patients with laceration in PITL level IIIA were treated conservative, because of stable clinical conditions.

Surgical approach was transcervical in 29 patients and thoracotomy in 14 patients. Differences in clinical parameters of these groups are shown in Tables 3,4.

Median length of injury was $4 \mathrm{~cm}$, ranging from 1 to $10 \mathrm{~cm}$. Thirty-seven patients had a laceration with a length up to $4 \mathrm{~cm}$ and 27 patients with more than $4 \mathrm{~cm}$. Thirty patients with a laceration up to $4 \mathrm{~cm}$ and 24 patients with an injury longer than $4 \mathrm{~cm}$ survived $(\mathrm{P}=0.498)$.

Fifty-five patients presented with mediastinal emphysema $(85.9 \%)$ and 43 additionally showed different levels of cutaneous emphysema (67.2\%).

There were several severe comorbidities in our cohort. Twenty patients presented with mediastinitis at admission $(31.3 \%)$, including 10 patients who had developed sepsis already (15.6\%). Patients' signs and symptoms according to survival are presented in Table 5 .

Thirteen patients had severe cardiologic diseases, including two cases of ST-elevational myocardial infarction, which were the underlying cause for emergency intubation. Eighteen patients were intubated because of respiratory insufficiency following chronic obstructive pulmonary disease and one patient with respiratory insufficiency following destroyed lobe caused by tuberculosis. One patient had an emergency intubation due to seizure. Fourteen patients suffered from primary lung cancer. 
Table 3 Differences in patients characteristics of individuals treated with cervical approach compared to thoracotomy

\begin{tabular}{|c|c|c|c|}
\hline Variable & Cervical approach $(n=29)$ & Thoracotomy $(n=14)$ & $P$ value \\
\hline Hospitalisation (days) & $14.21(29.78)$ & $10(6.92)$ & 0.607 \\
\hline BMI $\left(\mathrm{kg} / \mathrm{m}^{2}\right)$ & $28(4.39)$ & $28.5(2.93)$ & 0.702 \\
\hline Length of injury (cm) & $4.62(1.24)$ & $5(1.62)$ & 0.399 \\
\hline Pneumonia at admission (n, \%) & $13(44.83)$ & $8(57.14)$ & 0.526 \\
\hline Mediastinitis at admission (n, \%) & $11(37.93)$ & $9(64.29)$ & 0.191 \\
\hline Sepsis at admission (n, \%) & $5(17.24)$ & $5(35.71)$ & 0.252 \\
\hline CRP before treatment (mg/dL) & $6.36(6.13)$ & $11.24(8.17)$ & 0.034 \\
\hline In-hospital mortality (n, \%) & $5(17.24)$ & $5(35.71)$ & 0.252 \\
\hline Kruskal-Wallis test by ranks for PITL level & & & 0.109 \\
\hline PITL level IIIA (n, \%) & $18(78.26)$ & $5(21.74)$ & \\
\hline PITL level IIIB (n, \%) & $11(55.00)$ & $9(45.00)$ & \\
\hline
\end{tabular}

BMI, body mass index; CRP, C-reactive protein.

Table 4 Differences in patients characteristics of individuals treated with by bronchoscopy or surgical

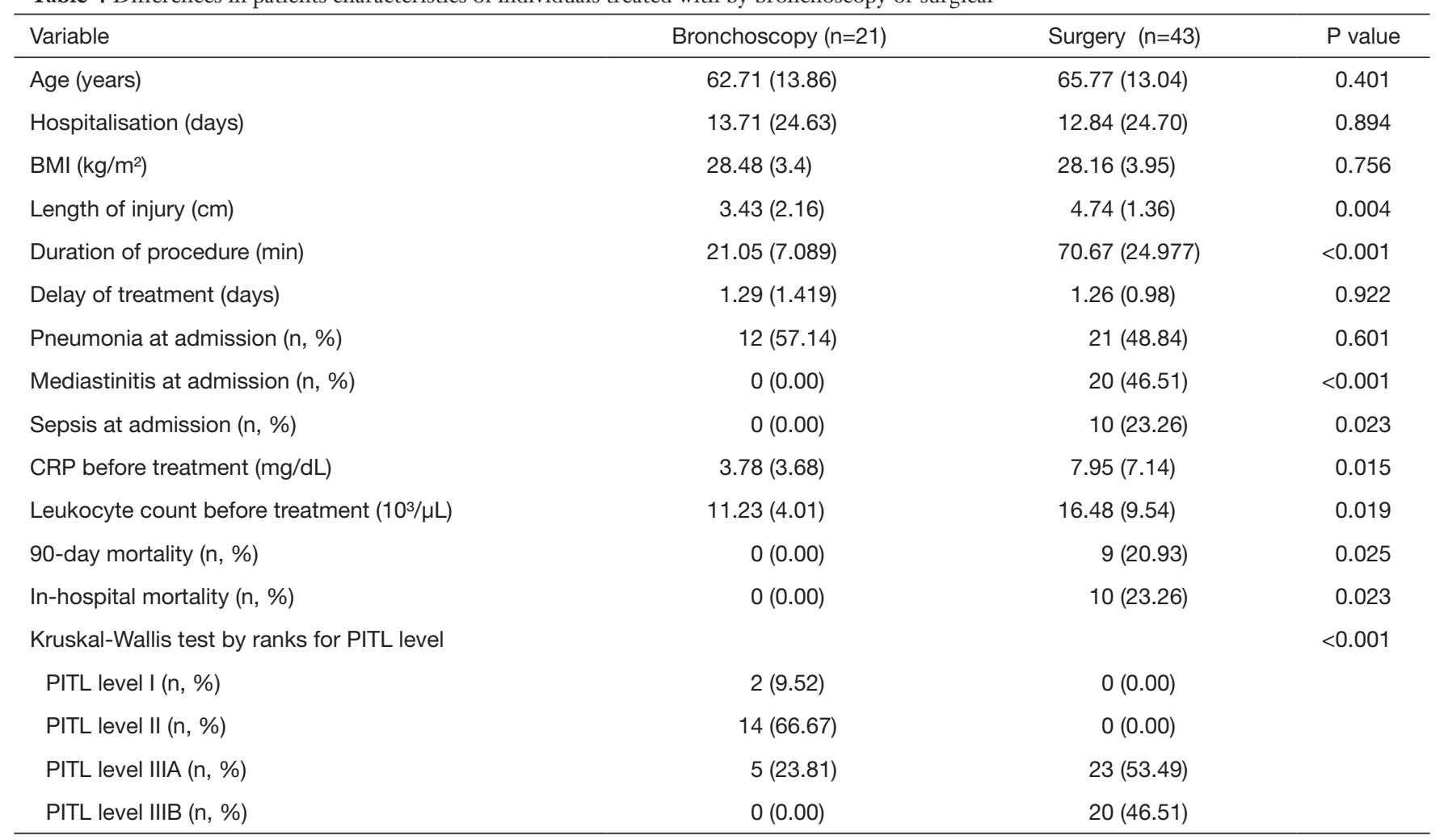

BMI, body mass index; CRP, C-reactive protein. 
Table 5 Patients' symptoms and correlation to overall survival

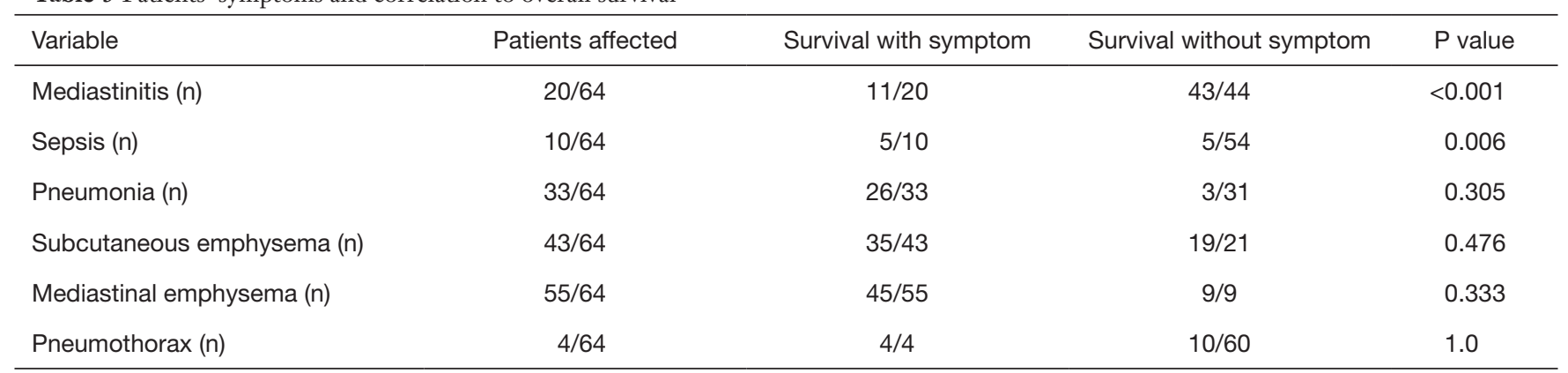

Thirty-three patients presented with pneumonia at time of intervention, 21 of them in the surgical group.

Sixty-two patients underwent a successful intervention; two patients died directly after surgery because of low cardiac output failure after finished reconstruction. None of the surgical patients needed revision.

Forty-two patients needed mechanical ventilation (65.6\%) before intervention. Two patients underwent extracorporeal membrane oxygenation for surgery in PITL stadium III.

The 30 - and 90 -day postoperative mortality was $14.1 \%$ $(n=9)$. Eight of these patients had been referred to our institution. All of them showed signs of mediastinitis and six of them presented with sepsis at admission already. The mean duration between occurrence of tracheal injury and referral of these patients to our unit was 1 day (range, $0-5$ days).

Two patients died of sepsis-associated multi organ dysfunction syndrome (MODS) during surgery; cardiopulmonary resuscitation had to be performed at onset of the operation. In both cases, a thoracotomy was performed due to PITL IIIB and laceration beginning in the distal third of trachea and ending in the right main bronchus.

Seven patients survived the operation but died in the following days with sepsis with MODS. These patients showed a median survival of 10 days (range, 1-14 days).

Another patient underwent a lobectomy of right lower lobe because of non-small cell lung cancer in our institution, with tracheal laceration PITL IIIA during elective doublelumen intubation. Following initially successful surgery for tracheal injury the patient died after 133 days due to severe respiratory insufficiency.

All patients treated conservatively with bronchoscopy survived.

\section{Discussion}

Tracheal injury is a rare but life-threatening complication after endobronchial intubation (11).

In this survey, we describe the standardized management of iatrogenic tracheal laceration in our thoracic surgery tertiary referral center with a focus on the results depending on surgical approach. We propose a treatment algorithm, based on the PITL-classification and necessity of mechanical ventilation.

The main findings of the study are:

(I) There is no difference in survival depending on surgical approach;

(II) Sepsis and mediastinitis are the only signs correlating with mortality;

(III) There is no difference in survival depending on length of the injury.

In stable patients without need for mechanical ventilation conservative management is an appropriate treatment. Indeed, in patients with transmural injury or necessity of mechanical ventilation surgical management of iatrogenic tracheal lesions is still the mainstay of treatment (11) and depending on the site of the laceration and the experience of the surgeon several approaches are available $(12,13)$. In 1995 Angelillo-Mackinlay presented repair of a tracheal lesion via a transcervical approach (9) and during the last decades cervical procedure became the standard for treatment of lesions of proximal two thirds of the trachea (11) to avoid the need of a thoracotomy and cause less trauma, in the intention to decrease the high mortality in these patients.

Several authors published reports about management of iatrogenic tracheal laceration including surgical treatment with up to 29 patients $(4,14-16)$, but only few describe differences of the outcome according to surgical approach.

Schneider et al. (4) reported a mortality of $16.7 \%(3 / 18$ patients) in the surgery group of their survey, but did not focus on differences in the surgical approach. Whereas Carbognani et al. (15) or Mussi et al. (16) presented cohorts of 13 , respective eleven patients and in both groups no 
patient deceased after surgical approach. Carbognani et al. treated six patients via thoracotomy and four patients via cervical approach, depending on the site of laceration. They decided for conservative management in three patients. While there was no mortality in their group they report a shorter length of stay for patients being treated with transcervical procedure compared to thoracotomy. Mussi et al. treated eleven patients with iatrogenic tracheal lesion. In their cohort two patients received conservative management, in four patients tracheal split was operated via thoracotomy and in five patients via cervical incision. There was no morbidity or mortality in their group and they profess shorter time of surgery for transcervical procedure. We are able to confirm these results, regarding the duration of the procedure. Mean duration of transcervial approach was $61.79 \mathrm{~min}$ in our cohort and thoracotomy lasted $89.07 \min (\mathrm{P}=0.001)$.

In another publication Lee and colleagues presented results of the treatment of eleven patients and nine of them underwent surgery. Four patients underwent cervical approach and five received thoracotomy. None of the patients who underwent surgery died; unfortunately, Lee et al. (17) did not focus on differences according to surgical procedure either.

In our cohort, consisting of 43 patients treated with surgery, 29 underwent cervical incision and transtracheal management for the tracheal lesion. There was no significant difference compared to 14 patients treated with thoracotomy related to survival, length of stay or infectious comorbidities. Despite presenting no difference in the outcome between both procedures, cervical approach is a feasible and safe procedure for management of iatrogenic tracheal lesions and furthermore the surgical trauma of a thoracotomy is not justified in these patients.

Iatrogenic tracheal laceration is a complication accompanied with a high mortality, which is stated with up to $42 \%$ (6). In most publications the underlying diseases are supposed to be the reason for the high mortality $(5,6,8,12)$. We present a cohort with a 90 -day-mortality of $14.1 \%$ and an in-hospital-mortality of $15.6 \%$. Of the investigated comorbidities, just mediastinitis and sepsis show a significant correlation to mortality.

Many authors propose mediastinitis as an indication for surgical management of tracheal laceration $(10,11,17)$ but only few studies focus on infectious comorbidities in their cohort (17-19).

Leinung and colleagues presented a survey with 42 patients treated for tracheal tear (19), 16 patients of their cohort died, ten of them due to infectious diseases. There were seven patients with mediastinitis at admission. Five of them underwent surgery and two were treated conservative. Surgery was not possible in these two patients, because they suffered from severe systemic inflammation. Both conservatively treated patients died and four of the five surgical treated patients experienced a suture insufficiency and needed revision. One of them died due to sepsis. In the whole cohort ten of the 16 deceased patients died because of sepsis. The authors present mediastinitis as a prognostic factor for suture insufficiency.

In another publication Gómez-Caro Andrés et al. presented experiences in management of iatrogenic tracheobronchial injury in 18 patients (18), 17 were treated conservatively and one patient underwent surgery. Four patients of this cohort died due to MODS following pneumonia, mediastinitis or peritonitis. None of the patients deceased because of tracheal injury related causes.

However, MODS is one of the most important complication and cause of death after interventions for iatrogenic tracheal lesion.

Several authors propose length of the tracheal tear being an indication for the choice of management $(12,14)$. There is a significant difference of the length of tracheal lesion in our conservative group compared to the surgical treated patients ( 3.43 vs. $4.74 \mathrm{~cm}, \mathrm{P}=0.004)$, but this was not relevant in the decision of management options. According to classification of Cardillo we took the depth of the tracheobronchial injury into account and neglected the length. In our cohort the longest laceration was a PITL II with a length of $10 \mathrm{~cm}$. This injury was treated successfully by application of fibrin sealant. Carbognani et al. postulate conservative treatment in case of an injury shorter than $2 \mathrm{~cm}$ (14). From this point of view only one patient in our group should have received conservative treatment. All in all, in our opinion the length of an injury is no reasonable parameter for management decision. The proposals in literature vary greatly and there is no evidence for any of them.

In recent years, several publications supported stent implantation to bridge a tracheal tear $(20,21)$. These case reports illustrate successful treatment in single patient or small groups and up today there is no evidence that this management is successful in non-selected patients.

Only few authors report their experiences of predominantly conservative management in groups with more than 30 patients $(8,22)$.

Tazi-Mezalek et al. present their experiences in 
management of tracheal laceration in 35 patients (8), where treatment decision was based on requirement of mechanical ventilation and site of laceration. Patients without mechanical ventilation were treated conservatively. Actually, only seven of the 35 patients received endotracheal stenting, because of tracheal tear in the lower half of trachea. In 17 patients the laceration was bridged by endotracheal tube or tracheotomy cannula and surgery was performed in case of tracheoesophageal fistula. Four patients of this series died. Three of these patients required mechanical ventilation and two of them were treated by stenting the laceration, which corresponds with a mortality rate of $29 \%$ in the group of patients treated with stents, which is as high as most reported mortalities in studies with predominantly surgical management (3). The authors suggest that the underlying medical condition was the reason for decease.

Comparing the results of Tazi-Mezalek et al. with our own cohort, they seem to be equivalent. The advantages, expected in use of endotracheal stents, are mainly based on case reports and criteria for the choice of using stents instead of surgical intervention are vague. Geltner et al. (23) report a case of a 64-year old woman suffering from iatrogenic tracheal tear with a length of $5 \mathrm{~cm}$ treated with endotracheal stent placement. Her symptoms were chest pain and dyspnea and CT shows a pneumomediastinum. Compared to Tazi-Mezalek et al. or our own recommendations conservative management and bronchoscopic surveillance might have been adequate treatment in this case and it is no prove for the superiority of management of this tracheal laceration with endotracheal stenting.

Furthermore, Tazi-Mezalek et al. state esophageal protrusion not being an indication for surgical intervention and that there is no benefit in tracheal stabilisation by surgery. Whereas Welter reports cases of expiratory tracheal collapse following mediastinal herniation after inadequate treatment leading to respiratory insufficiency (13). Another disadvantage of tracheal stenting is the possibility of distending the laceration with the stent, thus hindering healing in normal diameter(13).

Certainly, our study has limitations. It is a retrospective single-center study and therefore susceptible to selection bias. Nevertheless, we are confident that management of iatrogenic tracheobronchial injury on the basis of PITL classification is feasible and secure.

Early introduction of every patient with tracheal tear in a specialized center is recommendable, because prompt evaluation of the injury and rapid treatment might prevent severe infection.

Several approaches of management seem to be equivalent. In case of transmural injury of the tracheal wall and mechanical ventilation we recommend surgical treatment. In patients with lesion of the trachea we suggest cervical transtracheal approach in assumption of less traumatic management.

\section{Acknowledgments}

None.

\section{Footnote}

Conflicts of Interest: The authors have no conflicts of interest to declare.

Ethical Statement: The authors are accountable for all aspects of the work in ensuring that questions related to the accuracy or integrity of any part of the work are appropriately investigated and resolved. Institutional Review Board was consulted. No approval was required. Written informed consent from the patients was waived due to retrospective nature of the study.

\section{References}

1. Borasio P, Ardissone F, Chiampo G. Post-intubation tracheal rupture. A report on ten cases. Eur J Cardiothorac Surg 1997;12:98-100.

2. Massard G, Rougé C, Dabbagh A, et al. Tracheobronchial lacerations after intubation and tracheostomy. Ann Thorac Surg 1996;61:1483-7.

3. Schneider T, Volz K, Dienemann H, et al. Incidence and treatment modalities of tracheobronchial injuries in Germany. Interact Cardiovasc Thorac Surg 2009;8:571-6 .

4. Schneider T, Storz K, Dienemann H, et al. Management of iatrogenic tracheobronchial injuries: a retrospective analysis of 29 cases. Ann Thorac Surg 2007;83:1960-4 .

5. Lee BE, Korst RJ. Successful Treatment of an Iatrogenic Tracheal Laceration With a Temporary PolyurethaneCoated Nitinol Stent. Ann Thorac Surg 2016;102:e11-2.

6. Hofmann HS, Rettig G, Radke J, et al. Iatrogenic ruptures of the tracheobronchial tree. Eur J Cardiothorac Surg 2002;21:649-52.

7. Hussein E, Pathak V, Shepherd RW, et al. Bronchoscopic management of iatrogenic tracheal laceration using polyurethane covered nitinol tracheal stents. J Trauma 
Acute Care Surg 2016;81:979-83.

8. Tazi-Mezalek R, Musani AI, Laroumagne S, et al. Airway stenting in the management of iatrogenic tracheal injuries: 10-Year experience. Respirology 2016;21:1452-8.

9. Angelillo-Mackinlay T. Transcervical repair of distal membranous tracheal laceration. Ann Thorac Surg 1995;59:531-2.

10. Cardillo G, Carbone L, Carleo F, et al. Tracheal lacerations after endotracheal intubation: a proposed morphological classification to guide non-surgical treatment. Eur J Cardiothorac Surg 2010;37:581-7.

11. Ludwig C, Riedel R, Schnell J, et al. Inhalation with Tobramycin to improve healing of tracheobronchial reconstruction. Eur J Cardiothorac Surg 2009;35:797-800; discussion 800.

12. Grewal HS, Dangayach NS, Ahmad U, et al. Treatment of Tracheobronchial Injuries: A Contemporary Review. Chest 2019; 155:595-604.

13. Madden BP. Evolutional trends in the management of tracheal and bronchial injuries. J Thorac Dis 2017;9:E67-70.

14. Welter S. Repair of tracheobronchial injuries. Thorac Surg Clin 2014;24:41-50.

15. Carbognani P, Bobbio A, Cattelani L, et al. Management of postintubation membranous tracheal rupture. Ann Thorac Surg 2004;77:406-9.

16. Mussi A, Ambrogi MC, Ribechini A, et al. Acute major

Cite this article as: Herrmann D, Volmerig J, Al-Turki A, Braun M, Herrmann A, Ewig S, Hecker E. Does less surgical trauma result in better outcome in management of iatrogenic tracheobronchial laceration? J Thorac Dis 2019;11(11):47724781. doi: $10.21037 /$ jtd.2019.10.22 airway injuries: clinical features and management. Eur J Cardiothorac Surg 2001;20: 46-51, discussion 51-2.

17. Lee SK, Kim DH, Lee SK, et al. Does surgical repair still have a role for iatrogenic tracheobronchial rupture? Clinical analysis of a thoracic surgeon's opinion. Ann Thorac Cardiovasc Surg 2016;22:348-53.

18. Gómez-Caro Andrés A, Moradiellos Díez FJ, Ausín Herrero P, et al. Successful conservative management in iatrogenic tracheobronchial injury. Ann Thorac Surg 2005;79:1872-8.

19. Leinung S, Möbius C, Hofmann HS, et al. Iatrogenic tracheobronchial ruptures - treatment and outcomes. Interact Cardiovasc Thorac Surg 2006;5:303-6.

20. Cassada DC, Munyikwa MP, Moniz MP, et al. Acute injuries of the trachea and major bronchi: importance of early diagnosis. Ann Thorac Surg 2000;69:1563-7.

21. Marchese R, Mercadante S, Paglino G, et al. Tracheal stent to repair tracheal laceration after a double-lumen intubation. Ann Thorac Surg 2012;94:1001-3.

22. Conti M, Pougeois M, Wurtz A, et al. Management of Postintubation Tracheobronchial Ruptures. Chest 2006;130:412-8.

23. Geltner C, Likar R, Hausegger K, et al. Management of Postintubational Tracheal Injury by Endoscopic Stent Placement: case Report and Review of the Literature. Thorac Cardiovasc Surg Rep 2016;5:8-12. 